\title{
A Description Profound Fusion Recommender Scheme based on Self-Chipper with Neural Communal Filtering
}

\author{
P. Ajitha, A. Sivasangari, Bevish Jinila
}

\begin{abstract}
The Web makes fabulous open entryways for associations to give tweaked online organizations to their customers. Recommender systems intend to normally make altered suggestions of things/organizations to customers (business or individual). Regardless of the way that recommender systems have been particularly inspected, there are up 'til now two challenges in the enhancement of a recommenderstructure,particularlyinauthenticworldB2Be-

services. In Proposed a recommendation framework utilizing the speedy scattering and information sharing limit of an extensive customer orchestrate. This framework actualized a GRS dependent on conclusion elements that considers these connections utilizing a brilliant loads lattice to drive the procedure.InGRSs, asuggestionistypicallyfiguredbyabasic collection strategy for individual data the proposed technique [described as the client driven recommender framework (CRS)] pursues the community oriented sifting (CF)rule howeverperformsdispersedandnearbylooksforcomparative neighbors over a client arrange so as to produce a suggestion list.
\end{abstract}

Keywords-Recommender systems, B2B e-services, GRS, Collaborative filtering.

\section{INTRODUCTION}

Web mining is the approach towards separating information from subset ofa master set and consolidating it into significant information. This information would then have the capacity to be used to assemble pay, cuts costs, or both. An item made with web mining as its essential theme should empower customers to separate data from different estimations or focuses, sort it, and diagram the associations recognized. Truthbe told, web mining is the path toward finding associations or models among numerous fields in colossal social databases. Web mining is extremely the noninsignificant system of perceiving: Valid, Novel, Potentially supportive and inevitably legitimate models in data. By and large web mining will when all is said in done be misinterpreted with terms like chasing and examination, the going with table understandsthequalificationobviously. Thisendeavorisa growth of one of the outstanding sub-classes of web Mining:- "Market Based Analysis (MBA)", a procedure that gives an outlook of thecustomer base. A market container is made out of the thing sets which are purchased in a single trek to the store. MBA on a very basic level attempts to find the association betweenthethingspurchasedinthisbushel.Asapublicizing

\footnotetext{
Revised Manuscript Received on 14 August, 2019.

P. Ajitha, Associate Professor, Dept of IT, Sathyabama Institute of , Science and Technology, Chennai, Tamilnadu, India

A.Sivasangari, Associate Professor, Dept of IT, Sathyabama Institute of, Science and Technology, Chennai, Tamilnadu, India

BevishJinila, Associate Professor, Dept of IT, Sathyabama Institute of, Science and Technology, Chennai, Tamilnadu, India
}

device it is used to mine out the consistent thing sets in an immense no of trades. Thus it is in like manner called "Visit Item-setMining".

With the ongoing unstable development of the measure of substance on the Internet, it has turned out to be progressively troublesome for clients to discover and use data and for substance suppliers to arrange and list records. Customary web crawlers frequently return hundreds or thousands of results for a hunt, which is tedious for clients to peruse. On-line libraries, web search tools, and other substantial report storehouses (for example client bolster databases, item determination databases, official statement chronicles, news story files, and so on.) are developing so quickly that it is troublesome and exorbitant to order each report physically. So as to manage these issues, a look towards mechanized techniques for working with web archives so they can be all the more effectively perused, composed, and recorded with negligible human mediation. Instead of the exceedingly composed prohibited data whereupon most machine learning systems are depended upontowork, webandsubstancedocumentsaresemi-sorted out. Web records have especially described structures, for instance, letters, words, sentences, sections, zones, complement marks, HTML names, and so on. It is assessed that as much as $85 \%$ of all computerized business data, its greater part web-related, is put away in non-organized arrangements (ie .non-unthinkable configurations, for example, those that are utilized in databases and spreadsheets).

Making improved systems for performing machine learning techniques on this immense proportion ofnonunimaginable, semi-sorted out web data is thusly extremely appealing [15]. Bunching and characterization have been valuable and dynamic zones of machine learning research that guarantee to enable us to adapt to the issue of GraphTheoretic Techniques for Web Content Mining data overburdenontheInternet.Withpackingthegoal istosecludea given social event of data things into get-togethers considered gatherings to such a degree, to the point that things in a comparable gathering resemble each other and not at all like the things in various clusters. In bunching techniques no marked precedents are given ahead of time to preparing (this is called unsupervised learning). Under course of action we try to dole out a data thing to a predefined characterization reliant on an evolving model using already sortedtraining data (directed learning). From progressively broad terms, 


\section{A DESCRIPTION PROFOUND FUSION RECOMMENDER SCHEME BASED ON SELF-CHIPPER WITH NEURAL COMMUNAL FILTERING}

both bunching and arrangement go under the subject matter disclosure in databases or information mining. Applying information mining strategies to site page content is alluded to as mining of web content, a child field of mining in the area of data retrieval. While speaking to content and web record content for bunching and characterization, vector space is used

Inthismodel,everyconceivabletermthat can show up in a record turns into a component measurement. archive may demonstrate the occasions the comparing term shows up on it or it might be a load that considers other recurrence data, for example, the quantity of reports whereupon the terms show up. This model is elementary and permits the utilization of conventional ML strategies negotiating in and Euclidean element space with numerical element vectors. Be that as it may, it disposes of data, for example, the request in which the terms show up, where in the record the terms show up, how shut the terms are,etc. could enhance the execution of different machine learning calculations.

The issue is that conventional information mining strategies are frequently confined to chipping away at absolutely numeric element vectors because of the need to figure separates between information things or to compute some agent of a bunch of things, the two of which are effectively cultivated in an Euclidean space. Thusly either the principal data ought to be changed over to a vector of numeric characteristics by discarding maybe important assistant information or we need to become new, changed techniques for the specific depiction. This endeavor intends toaccomplishastreamlinedpredictingfiguringtowatchthe ceaseless things obligated to be procured by the customer. Here we look at the past gaining instances of the customers and use the information thus anchored, to connectidentified with the purchasing attitude of explicit plans of customers. Association structures among things inside an E-business Web site page can be seen as a potential recommendation that helps new purchasers quickly find critical things. We proposeasuggestionstrategyusingthequickdispersionand data sharing capacity of a substantial clientarrange.

\section{RELATEDWORKS}

1.Jiawei Lu, Yu Guo, Yang Yang, ZhenqiangMi, 2017. Because of its straight forwardness and adequacy, communitariansifting $(\mathrm{CF})$ time is the most widely used suggestion measurement. CF's two main classes have their own weaknesses. With information sparsity, memory-based CF can not create; and model-based CF reliably loses data between customers or things. To explain this problem, we propose a calculation that integrates consumer trust in the traditional factorization system (MF). Trust coordinate knows how to use all the customers trusted to help make predictions. Analyzes were carried out on the database Epinions and Movie Trust to compare the methods proposed. The detailed outcomes show that utilizing client trust into $\mathrm{MF}$ is legitimate and can enhance the suggestionquality.

2.Greg Linden, Brent Smith, and Jeremy York, 2003. Suggestion calculations are best known for their utilization onwebbasedbusinessWebdestinations, wheretheyusecontribut ion about a client's advantages to produce a rundownofprescribedthings.Different systems only use the products that customers buy and value to answer their preferences honestly. We can use distinguishing attributes similarly, like items observed, measurement information, topic interests, and most beloved experts. We use recommendation formulas at Amazon.com to tailor the online store for each customer. The shop shifts radically based on consumer desires, showing a brand architect's system titles and another mother's baby toys. The level of navigation and adjustment two important proportions of Web-based and email fostering adequacy exceed tremendously those of untargeted content, such as pennant ads and best merchant records.

3.Y. Koren, R. Bell, and C. Volinsky, 2009. Purchasers of the present day are immersed in decisions. Electronic retailers and distributors of materials offer a huge selection of items, with excellent opportunities to meet a variety of unique needs and tastes. To order to improve customer satisfaction and steadfastness, connecting consumers with the most appropriate things is important. Further retailers have turned out to be keen on proposing mechanisms along these lines, breaking down examples of consumer interest for products to provide personalized recommendations that match the taste of a customer. Since good personalized solutions could add another dimension to consumer experience, web-based business giants such as Amazon.com and Netflix have made recommender systems a prominent feature of their pages.

4. P.Vincent,H.Larochelle,Y.Bengio, andP.A.Manzagol, 2009. Previous work has shown that the complexities of adapting deep generative or discriminative models can be exacerbated by an underlying unsupervised learning phase mapping inputs to helpful halfway portrayals. The planning guideline for unsupervised training of a representation is introduced and convinced to make the informed portrayals vigorous to the fractional degradation of the data model.This technique can be used to prepare auto-encoders, and these opposing auto-encoders can be stacked in order to introduce deep designs. The calculation can be roused from a complex theoretical point of view of learning and information, or from a point of view of the generative method. Close analyzes show unmistakably the stounding favorable position of the auto-encoders ' contribution to an example order benchmark set.

5.RuiningHe,JulianMcAuley,2016.Current recommender systems reveal individuals and objects by seeking the simple measures that represent the properties of the inclinations of things and clients towards them or' prodding separately.' Essentially, these measurements are disclosed based on consumer feedback, periodically in understood form (e.g. purchasing accounts, using logs, etc.).

However, some systems of recommenders make use of side data, such as item properties, fleeting information, or survey material.Nevertheless, the visual appearance of the items being considered is a vital component that is routinely overlooked by current personalized proposal and positioning techniques. A flexible method of factorization to integrate visual signsinto indicators of feelings of individuals that we

Blue Eyes Intelligence Engineering

\& Sciences Publication

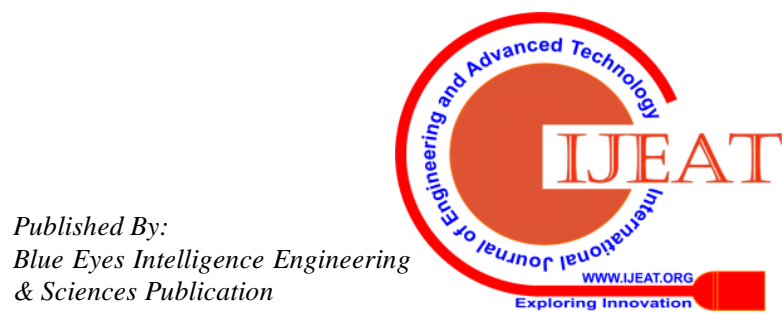


apply to evaluating vast, genuine world datasets.Using (preprepared) deep structures, they make use of visual highlights isolated from item images, over which we become acquainted with an extra layer which exposes visual dimensions that better explain the variety of input by individuals. This not only encourages personalized positioning techniques to be completely accurate, but also eliminates issues with cold starting and subjectively examines the visual indicators that affect the expectations of individuals.

6.Matthew D. Zeiler and Rob Fergus, 2016. Huge Convolution Network models have shown such a spectacular execution of characterization on the Krizhevsky et al Image Net test. Nonetheless, there is no fair idea as to why they do so well and how they can be pushed forward. We are investigating the two problems in this journal. We present a new representation system that provides information about the potential of moderate component layers and the classifier's mission.UsedIn a symptomatic job, these representations help us to discover on the Image Net grouping benchmark display models that beat Krizhevsky et al. We also play a removal focus in order to find the dedication to execution from different model surfaces. We prove our Image Net model aggregates well to different data sets: it convincingly beats the latest bleeding edge findings on Caltech-101 and Caltech-256 datasets when the soft max classifier is retrained.

7.AlexKrizhevsky, IlyaSutskever, Geoffrey E. Hinton, 2016. We prepared a massive, deep neural convolution model to characterize the 1,2 million high-goal objects in the LSVRC-2010 Image Net challenge into the 1000 distinct classes. We achieved best 1 and best5 screw-up rates of 37.5 percent and 17.0 percent on the test data, which stands out remarkably better than the past.[14] The neural framework, which has 60 million parameters and 650,000 neurons, contains five layers of convolution, some of which are trailed through max-pooling layers, and three fully connected layers with a last 1000-way soft max. We used non-immersing neurons and a successful GPU use of the convolution process to make training faster. We used a latecreated regularization technique called "dropout" to minimize overfitting in the fully linked layers which ended up being convincing. Furthermore, in the ILSVRC-2012 competition, we joined a variant of this template and obtained a victorious best 5 test blunder rate of 15.3 percent, compared to 26.2 percent of the consistently best passage.

8.XiangnanHe, TaoChen,Min-

YenKan,XiaoChen,2015.Many current group ltering approaches have concentrated on illustrating the parallel link between consumers and issues by distinguishing them from consumer assessments

Besides the evaluations of customers, their related reviews also give their assessments the logical method and consider the aspects of the issue they often think about. To enhance the top- $\mathrm{N}$ suggestion, we investigate the rich proof wellspring of angles in client surveys.Through excluding angles (i.e., the specific properties of things) from literary audits, they advance the double relation of the client thing to the ternary connection viewpoint of a client thing. As a heterogeneous tripartite graph, we show the ternary relation, giving the proposal assignment a function as one of vertex positioning. We layout a traditional positioning calculation for TriRank trip artite charts and practice it for custom proposal. Trials on two open data sets of surveys show that it consistently outstrips cutting-edge approaches. Above all, by demonstrating angles in surveys, TriRank blesses the recommender framework with a higher level of clarification capacity and simplicity. This allows clients to interact from their point of view inclinations with the system, helping clients to decide on informed choices.

9.SteffenRendle, 2001. A Factorization Machines (FM) that are another model class that combines the benefits of Support Vector Machines (SVM) with factorization models. Unlike SVMs, FMs are a general indicator that operates with any truly valued vector of components. FMs display all correlations between variables using factorized parameters as opposed to SVMs. [13] Accordingly, even in issues of enormous sparsity (such as proposing frameworks) where SVMs fall flat, they may evaluate cooperation.We demonstrate that the design state of FMs can be calculated in a straight time and FMs can be updated directly along these lines. Therefore, a change in the double shape is not critical at all like nonlinear SVMs, and the design parameters can be evaluated directly without the need for any support vector in the arrangement.

10.SteffenRendle, ChristophFreudenthaler, Zeno Gantner and Lars Schmidt-Thieme 2001. Thing suggestion is the assignment on a lot of things to anticipate a custom positioning. With some feedback (e.g. clicks, purchases), they discuss the most widely recognized scenario. From verifiable feedback such as system factorization (MF) or flexible k-closest neighbor $(\mathrm{KNN})$, there are various recommendation techniques. Although these techniques are intended for the personalized positioning errand of the thing forecast, none of them is updated specifically for positioning.[12] present a traditional BPR-Opt streamlining basis for custom positioning which is the largest back estimator obtained from a Bayesian problem analysis. We also offer advancing models as for BPR-Opt a traditional learning estimate. The learning technique relies on bootstrap-examining stochastic slope plunge. They say the best way to apply our technique to two recommender models of cutting edge: factorization of lattice and scalable $\mathrm{KNN}$.

11.This is intended to improve the accessibility of numerous reviews to be viewed as the final review edition. This also offers a more efficient way to predict and thus imply the user's view.

\section{SYSTEMARCHITECTURE\& RESULTS}

Themodulesoftheundertakingalongside themannerinwhichtheyareexecutedandthatisarrangedas for the proposed framework, while conquering existing framework and furthermore giving the help to the future improvement framework. There are absolutely fivemodules utilized in our venture which is recorded underneath. Every modulehasexplicituseintheundertakingandisportrayalis given underneath pursued by the rundown ofmodules. 


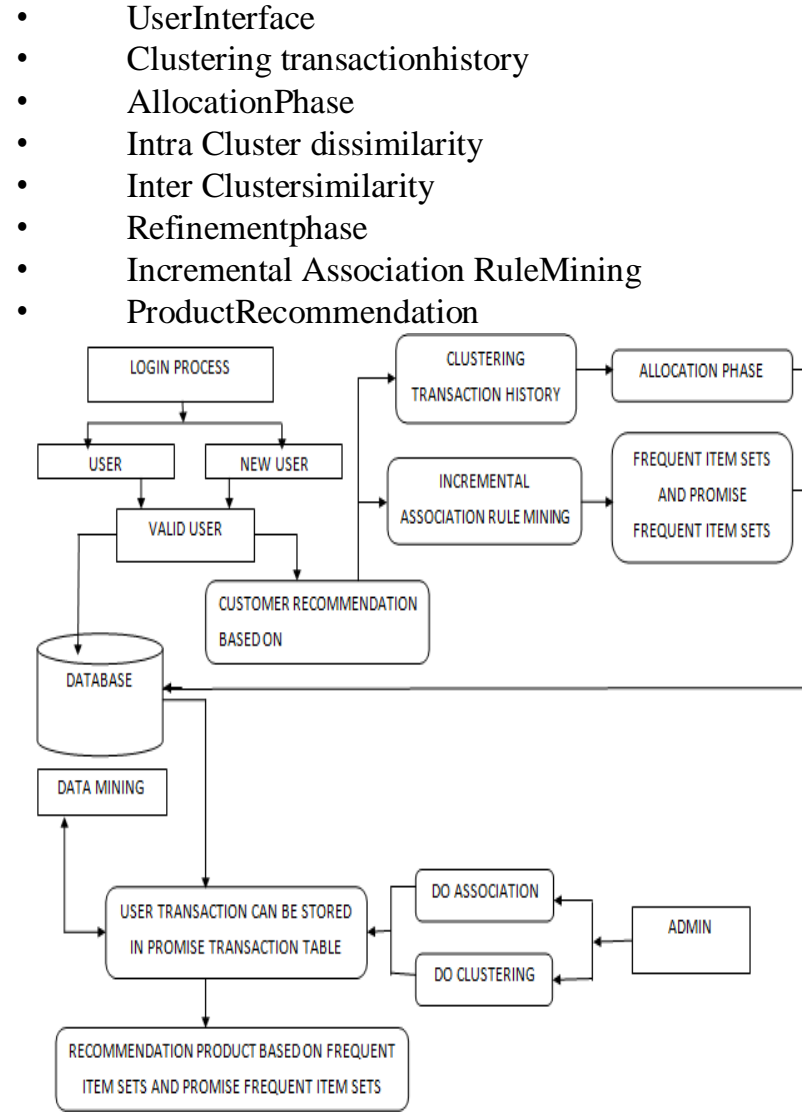

Fig.3.1 System Architecture Design

\section{A. USERINTERFACE}

In the modern structure field of human- machine connection assumes a vital job. It is where communication among people and machines happens. Its objective of collaboration between a human and a machine at the UI is viable activity. Info enabling the clients to control a framework. The client will perform either login or enlistment task. After this activity get over he will go to the following stage.

\section{B. CLUSTERING TRANSACTION HISTORY}

The initial phase in the search process is to cluster the transaction history server. The account history file contains the customers ' past transactions. Customer I d, the collection of items purchased along with the transaction I dare included in the data.

\section{ALLOCATIONPHASE}

Organizing in the segment; in the game plan, each trade is examined. Any exchange can be allocated to an existing group or another bunch will be made to compel t to limit allout grouping expenses. InDB is reframed for each exchange at the first designated bunch lookup-id. The inclusion of the transaction in the current or newly formed clusters is decided on the basis of the clustering.

\section{INTRA-CLUSTER DISSIMILARITY}

Intra-aggregate divergence shows us how phenomenal the trades are within a bundle. $\operatorname{Intra}(U)=\operatorname{sm}(C j, E) \operatorname{Intra}(U)-$ Intra-bundle difference $\mathrm{Sm}$ small things $\mathrm{Cj}-\mathrm{j}$ th group $\mathrm{E}$-the most extreme roof. The most severe roof is the largest number of exchanges that could hold something to call a small thing. Therefore, the intra-aggregate gap tends to be the main relationship.

\section{E. INTER-CLUSTER SIMILARITY}

The value of the cluster decides the similarity between clusters. The total cost is assessed as Cost $=\mathrm{W}^{*}$ Intra(U)+Inter(U) where w-Itight Intra(U) -Inter(U) -Intercluster similarity within the cluster. The exchange is put in each of the current bunches for the first time, and the value for each party is determined. Another bunch is made at that point to suit the exchange and the costs. Eventually, the trade is assigned to the batch with the lowest cost of a reward. Compute cost Compare the cost to the best cost to date If (new expense is better) Assign current expense to best cost Assign current bunch to best group. Create another category for the current exchange Calculate price. Contrast the cost so far with the best cost. In case that is better (new expense). Dole to better cost the present bill. Get the best bunch out of the current group.

\section{F. F.REFINEMENTPHASE}

The small massive proportion (SL ratio) of the considerable number of exchanges is calculated as followed in the refinement process. $\mathrm{SLR}=$ (no. of small things)/(no. of big things) The SL ratio of each exchange in this mannerdeterminedisthencontrastedandtheSLRlimit. On the unlikely chance that the exchange SLR reaches the limit, the exchanges are moved to the abundance pool. The effort is then made to oblige these exchanges to be an alternative set, if the new group's SLR of these exchanges does not reach the bottom. Exceptions are perceived and washed out of mind if not such exchanges.

\section{G. INCREMENTAL ASSOCIATION RULEMINING}

The list of trade documents contains the customers ' past transactions. The subtleties include customer $\mathrm{I} d$, the arrangement of purchased items alongside the exchange I d. There are two sub-phases in this stage,

1. Original databaseDiscovery

2. Updating regularly, promising itemsets.

\section{ORIGINAL DATABASE DISCOVERY}

A dynamic database can allow new exchanges to be embedded. This can deny current managers of affiliation as well as enact new rules of affiliation. A critical issue is the preservation of affiliation rules for a complex server. Another calculation is therefore proposed to manage such refreshing circumstance. The reason for the new estimate is that new exchange rates are gradually changing from one-off transactions. The measures of older changes gained from past mining, as per the supposition, can be used to estimate that of new exchanges. Subsequently, in the wake of embedding the new exchanges in a specific database containing old exchanges, the support check of things acquired from past mining that set some are not quite the same as aid tally of stuff.

The latest calculation uses the most extreme support test that 1-itemsets have been collected from past mining to gage 
rare thing sets of a specific database that is designed to be successive sets once new exchanges are inserted in the first database. With the greatest support check and the most severe size of new exchanges that require integration into a common database, bolster means inconsistent sets of items that fit the bill for incessant sets of things, i.e. manipulating, are shown in equation 1 :

Min_sup-((max_supp/total size $) *$ inc_size $)<=$ min_PL $<\min \_$sup $(1)$

Where min sup (DB) is the minimum amount of support for an original server, mix-up is the maximum number of element sets of support. In this paper, the calculation of apriority is connected to locate all conceivable successive kthing sets and guarantee continuous k-thing sets. Apriority filters all exchanges of a common server are joining and prune phase for each focus with 2-day procedures. A mixture of Successive and incessant k-thing sets could be achieved, unlike usual apriority calculation.For a constant thing, its support test must be higher than a client's least help tally edge suggested, and for a promising incessant thing, its help count must be higher than manipulating yet not exactly the least helpful client decided.

\section{UPDATING REGULARLY, PROMISING ITEMSETS}

Once new exchanges are added to a different list, an old stable k-thing may turn into an erratic k-thing, and an old promising daily thing may turn into an incessant k-thing. It presents new criteria for membership, and some current membership standards will end up invalid.

All k-things need to be refreshed when new exchanges are added to a unique database to manage this issue. It reveals how every single old thing can be refreshed in this segment. Measurement of fluctuations in a revamped system if new transactions were inserted in a specific database. Min PL must be recalculated in this manner in order to connect to the new size of the freshed database. Min PL (update) shall be determined as follows:

Min_PL=min_sup-(max_supp/total size*inc_size) (2)

So, on the off chance that any k-thing will have a more worthwhile frequency test than min sup (DBUdb), moved to the next k-thing of a modified list. In the other case, it is not exactly min sup (DBUdb) if any k-thing has a bolster search. We also have to update a worthier entity or the same entity. While this k-thing is moved to a fresh server guarantee visit itemset. The following calculations were made to update the visit and guarantee an updated database's daily k-terms.

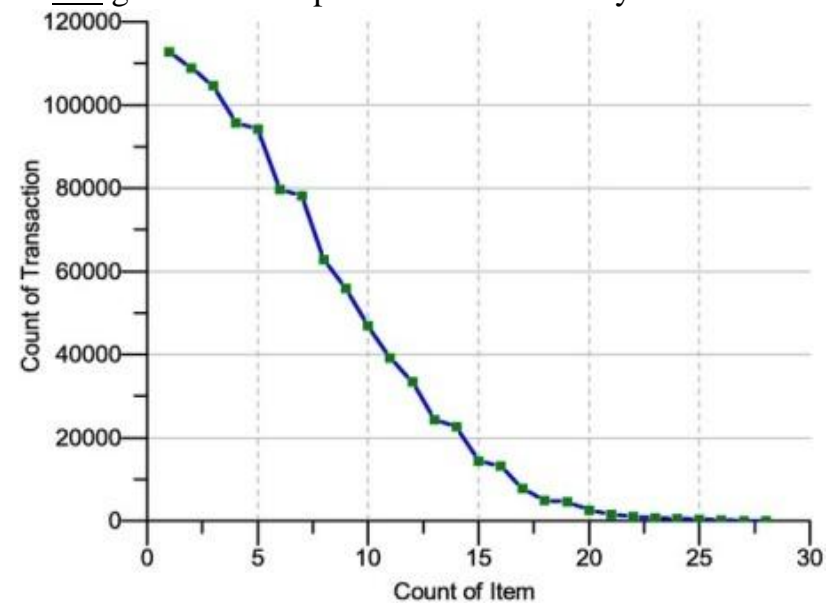

\section{CONCLUSION}

With the assistance of Incremental Association Rule Mining and Transaction Clustering, It acquainted a strategywithplananenhancedandallaroundorganizedweb composition for an E-shop in the structure stage. Expecting thatthetwolimits,leasthelpandcertainty,don'tchange,the promising successive calculation can ensure to find visit thing sets. It has utilized a productive bunching calculation for information things to limit the SL proportion in each gathering. The calculation can bunch the information things very productively. This calculation acquires an execution time as well as prompts the grouping consequences of extremely greatquality.

\section{REFERENCES}

1 J.W.Lu,Y.Guo,Z.Q.Mi,Y.Yang,Trust-enhanced matrix factorization using PageRank for recommender system, in Proc. 2017 Int. Conf. Computer, Information and TelecommunicationSystems,Dalian,China,2017,pp.123- 127.

2 G. Linden, B. Smith, and J. York, Amazon.com recommendations: Item-to-item collaborative filtering, IEEE Internet Compute., vol. 7, no. 1, pp. 76-80,2003.

3 Y. Koren, R. Bell, and C. Volinsky, Matrixfactorization techniquesforrecommendersystems, Computer,vol.42,no. 8, pp. 30 37,2009 .

4 P. Vincent, H. Larochelle, Y. Bengio, and P. A. Manzagol, Extracting and composing robust features with denoisingauto encoders, in Proc. 25th Int. Conf. Machine Learning, Helsinki, Finland, 2008, pp. 10961103

5 R. N. He and J. McAuley, VBPR: Visual Bayesian personalized ranking from implicit feedback, in Proc. $30^{\text {th }}$ AAAI Conf. Artificial Intelligence, Phoenix, AZ, USA, 2016, pp. 144-150.

6 M. D. Zeiler and R. Fergus, Visualizing and understanding convolutional networks, in Proc. European Conf.ComputerVision,Zurich,Switzerland,2014,pp.818-833.

7 A.Krizhevsky,I.Sutskever,andG.E.Hinton,ImageNet classification with deep convolutional neuralnetworks,in Proc. 25th Int. Conf. Neural Information Processing Systems, Lake Tahoe, NV, USA, 2012, pp. 1097-1105.

8 X. N. He, T. Chen, M. Y. Kan, and X. Chen, TriRank: Review-aware explainable recommendation by modeling aspects, in Proc. 24th ACM Int. Conf. Information and Knowledge Management, Melbourne, Australia, 2015, pp. 1661-1670.

9 S. Rendle, Factorization machines, in Proc. 10th Int. Conf. Data Mining, Sydney, Australia, 2010, pp.995-1000.

10 S.Rendle,C.Freudenthaler,Z.Gantner,andL.SchmidtThieme, BPR Bayesian personalized ranking from implicit feedback, in Proc. 25th Conf. Uncertainty in Artificial Intelligence, Montreal, Canada, 2009, pp.452-461

11 Jenitha Sri R, Ajitha P," Survey of product reviews using sentiment analysis",Indian Journal of Science and Technology, Vol 9(21), June 2016,PP1-4.

12 Nirmalrani V and Sakthivel P, “A Hybrid Access Control Model with Multilevel Authentication and Delegation to Protect the Distributed Resources", Journal of Pure and Applied Microbiology (JPAM), Vol. 9 (Spl. Edn. 2), pp. 595 - 609, November 2015

13 A.Sivasangari, P.Ajitha, K.Indira,"Air Pollution Monitoring and Prediction using Multi View Hybrid Model", International Journalof Engineering and Advanced Technology (IJEAT)ISSN: 2249 8958,Volume-8, Issue-2S,pp.1370-1372.

14 R.Subhashini and V. JawaharSenthil Kumar, "A Framework for Efficient Information Retrieval using NLP Techniques “, Proceedings of the International Conferences on Advances in Communication Network and Computing, CNC 2011, CCIS 142, pp. 391-393, 2011 , Springer-Verlag Berlin Heidelberg 2011,ACEEE, Bangalore.

15 Subhashini, R., Milani, V, "Implementing geographical information system to provide evident support for crime analysis", Procedia Computer Science, 2015 